\title{
Editorial
}

\section{Atropine and succinylcholine: beliefs and controversies in paediatric anaesthesia}

\begin{abstract}
Atropine was isolated in pure form in $1831 .^{1}$ One century and sixty-three years later, understanding of its complex pharmacology has yet to be achieved. Succinylcholine was introduced into clinical practice in $1951 .{ }^{2}$ Forty-three years later, we can only explain partly its mechanism of action at the neuromuscular junction, the duality of its neuromuscular block, and the diversity of its neurovegetative effects. Information on neuromuscular physiology, on neurohumoral transmission, and on the locations of different sub-types of cholinergic receptors remains fragmentary. ${ }^{3}$ In such a context, it is not surprising that issues of anticholinergics and succinylcholine in paediatric anaesthesia remain controversial.
\end{abstract}

Atropine elicits two opposite cardiac effects, according to the dose used: (a) slowing of the heart rate, following $1.8 \mu \mathrm{g} \cdot \mathrm{kg}^{-1} i v$ in adults, or $3.6 \mu \mathrm{g} \cdot \mathrm{kg}^{-1} i v$ in infants and children; and (b) acceleration of the heart rate, following a minimum of $7.2-14.3 \mu \mathrm{g} \cdot \mathrm{kg}^{-1} \dot{i}$ in infants, children, and adults. ${ }^{4}$ It was thought that atropine and scopolamine caused cardioinhibition by central vagal stimulation. ${ }^{5}$ However, cardiac slowing is also seen with uaternary antimuscarinics which hardly cross the bloodrain barrier (atropine methylbromide, glycopyrrolate, and pirenzepine). ${ }^{1,6-7}$ As a small dose of pirenzepine, an $\mathbf{M}_{1}$-antagonist, can abolish the cardioinhibitory effect of atropine, it is now realized that atropine and other nonselective antimuscarinics can also produce bradycardia by blocking peripheral $\mathrm{M}_{1}$-autoreceptors which normally inhibit or modulate the liberation of acetylcholine. ${ }^{7}$ Cardioacceleration results from blockade of $\mathbf{M}_{2}$-receptors on the SA nodal pacemaker. 'Intravenous atropine is commonly used in paediatric anaesthesia to (1) prevent and treat myocardial depression (bradycardia with hypotension) induced by halothane, (2) prevent and treat cardioinhibition associated with potent opioids (fentanyl and congeners), (3) reduce the incidence of the oculocardiac

From the Department of Anaesthesia, Hôpital Sainte-Justine, Université de Montréal. and other trigeminocardiac reflexes, (4) reduce the incidence of bradycardia resulting from airway manipulation (laryngoscopy, intubation) and visceral stimulation, (5) counteract the muscarinic effects of neostigmine or edrophonium, (6) reduce secretions during oropharyngeal surgery or when using ketamine, and (7) prevent bradycardia and bradyarrhythmias secondary to an intravenous bolus of succinylcholine. In each of these situations, atropine $i v$ is better used to prevent than to treat the effects of parasympathetic stimulation. If atropine is given during vagal stimulation, there is the risk of converting sinus bradycardia into premature ventricular contractions and runs of bigeminy. ${ }^{8}$ Furthermore, if atropine is given during bradycardia, particularly in infants, the cardiac output is reduced and the drug takes longer to produce its cardioaccelerating effect. ${ }^{9}$

Some authors believe that the dose of atropine for infants and children is $0.02 \mathrm{mg} \cdot \mathrm{kg}^{-1}$ (im or iv) and emphasize that the "minimum dose" for infants under one year is $0.1 \mathrm{mg}$. 10,11 The danger of this "simplification" is illustrated by the case report of a neonate who developed a toxic reaction (lethargy, opisthotonus, seizures, periodic breathing, dilated unresponsive pupils, dry mucous membranes and skin, and urinary retention) following two "minimum doses" of atropine $\left(0.09 \mathrm{mg} \cdot \mathrm{kg}^{-1}\right.$ $i v)$ over five hours. ${ }^{12}$ Although several children have survived accidental overdosage with large amounts of atropine (16-40 mg $\left.\cdot \mathrm{kg}^{-1} \mathrm{po}\right),{ }^{11,13-14}$ Gillick $^{12}$ underlines that death in children from atropine poisoning has occurred with doses as small as $0.05 \mathrm{mg} \cdot \mathrm{kg}^{-1}$. Wide individual variations have been reported in the pharmacokinetic $^{15}$ and pharmacodynamic behaviour of atropine. ${ }^{4}$ Age-dependent differences have been evidenced by Dauchot and Gravenstein ${ }^{4}$ using incremental doses of atropine in conscious patients. Atrial and high nodal rhythms, following $1.8-3.6 \mu \mathrm{g} \cdot \mathrm{kg}^{-1}$ atropine $\dot{i}$, are more frequent in children, while A-V dissociation is the most common cardiac arrhythmia in adults receiving 3.6-7.2 $\mu \mathrm{g} \cdot \mathrm{kg}^{-1}$ atropine $i v^{4}$ Pharmacokinetic studies have 
shown that younger children ( $<$ two yr) have an increased volume of distribution $\left(3.2 \mathrm{~L} \cdot \mathrm{kg}^{-1}\right)$ and a longer elimination phase half-life $(6.9 \mathrm{hr})$, following $0.01 \mathrm{mg} \cdot \mathrm{kg}^{-1}$ atropine $i v$, compared with older children $\left(1.3 \mathrm{~L} \cdot \mathrm{kg}^{-1}\right.$ and $2.5 \mathrm{hr}$, respectively), or with adults $\left(1.6-3 \mathrm{~L} \cdot \mathrm{kg}^{-1}\right.$ and 3-4 hr, respectively, according to the dose used: $\left.0.01-0.03 \mathrm{mg} \cdot \mathrm{kg}^{-1}\right){ }^{15-17}$ Ethnic differences have been identified in adults but not studied in paediatric populations. White Americans seem more prone than black Americans to develop bradycardia, following small doses of atropine. ${ }^{18}$ South-African Vendas seem more sensitive to the cardioinhibitory effect of atropine than white South-Africans. ${ }^{19}$ Chinese subjects seem much more sensitive to the cardioaccelerating effect of atropine than white Americans. ${ }^{20}$ Halothane, compared with enflurane, enhances the cardioaccelerating effect of anticholinergics in infants and children. ${ }^{21}$ Finally, one should remember than many $\mathrm{H}_{1}$-antihistaminics, phenothiazines, tricyclic antidepressants, gallamine, and pancuronium, have antimuscarinic activity which may justify a reduction of dose, or omission, of atropine.

In this issue, Shorten et al. ${ }^{22}$ show that it is not necessary to administer more than $10 \mu \mathrm{g} \cdot \mathrm{kg}^{-1}$ of atropine to older children (6-16 yr) before thiopentone (5 $\left.\mathrm{mg} \cdot \mathrm{kg}^{-1}\right)$ and succinylcholine $i v\left(1.5 \mathrm{mg} \cdot \mathrm{kg}^{-1}\right)$. One could argue that this study does not present unexpected results, but only confirms what has been suggested by others since 1963,,$^{23-25}$ and includes teenagers in a small selection of patients. Some anaesthetists will question whether the results justify a change in their habits. Some twenty years ago, we re-examined the problem of the "minimum effective dose" of atropine $i v$ in paediatric patients because we had noted that $0.02 \mathrm{mg} \cdot \mathrm{kg}^{-1}$ was often excessive as it caused a high incidence of side effects leading to postoperative discomfort (tachycardia, dry mouth, thirst, blush, erythematous rash over the neck and chest, hyperthermia, urinary retention, loss of accommodation, and agitation). Such side effects were particularly evident in younger children who had received two doses of atropine $\left(0.04 \mathrm{mg} \cdot \mathrm{kg}^{-1} i v\right)$ for the same anaesthesia (one preanaesthetic dose plus one dose in the reversal of curarization, for example). Consequently, we decided to titrate the chronotropic response of each patient by using incremental doses of $0.01 \mathrm{mg} \cdot \mathrm{kg}^{-1}$ of atropine $i v$. It did not take long to verify that a bolus of $0.01 \mathrm{mg} \cdot \mathrm{kg}^{-1}$ of atropine was remarkably effective in producing cardioacceleration and in reducing or abolishing the cardioinhibitory effects of halothane, succinylcholine iv (1-1.5 $\mathrm{mg} \cdot \mathrm{kg}^{-1}$, following thiopentone $6-8 \mathrm{mg} \cdot \mathrm{kg}^{-1}$ ), or vagal reflexes, while allowing a substantial reduction in postoperative discomfort. Since then, except for the reversal of curarization, we rarely use more than 10 $\mu \mathrm{g} \cdot \mathrm{kg}^{-1}$ of atropine $i$. Furthermore, cumulative dose- response curves for atropine $i v$ confirm that the "minimum effective dose" to produce cardioacceleration lies somewhere between 7.2 and $14.3 \mu \mathrm{g} \cdot \mathrm{kg}^{-1}$ in infants, children, and adults. ${ }^{4}$ Therefore we believe that Shorten $e t$ al. ${ }^{22}$ have found a reasonable dose of atropine $i v$ to prevent succinylcholine bradycardia.

Bradycardia $\left(<100\right.$ beats $\cdot \min ^{-1}$ in the first yr of life, 98 beats $\cdot \mathrm{min}^{-1}$ in the $2 \mathrm{nd}$ and $3 \mathrm{rd} \mathrm{yr}, 60-65$ beats $\cdot \mathrm{min}^{-1}$ in older children) is often a sign of hypoxia, severe hypotension, or anaesthetic overdosage, leading to cardiac arrest particularly in infants. Failure to recognize the presence and cause of bradycardia and failure to prevent or reverse the trend in slowing of the heart rate seem to be more frequent with novice than with experienced paediatric anaesthetists. ${ }^{26,27}$

Succinylcholine, when not contraindicated, is the muscle relaxant of choice for rapid-sequence induction, laryngospasm, rapid re-intubation, and intramuscular administration. ${ }^{28-30}$ Consisting of two molecules of acetylcholine linked together, succinylcholine may reproduce every effect of acetylcholine at both nicotinic and muscarinic receptors. In nonatropinized children (three months to seven years), well-ventilated and anaesthetized with halothane $(\mathrm{H}), 2.5 \%$ in oxygen, or isoflurane (I), $3.5 \%$ in oxygen, a bolus of succinylcholine iv (1.5 $\mathrm{mg} \cdot \mathrm{kg}^{-1}$ ) elicits three different and unpredictable responses in the heart rate: (a) increase (incidence of $66 \%$ with $\mathrm{H}, 88 \%$ with $\mathrm{I})$, (b) no change $(0-7 \%, \mathrm{I}-\mathrm{H})$, or (c) decrease $(12-27 \%$, I- $\mathrm{H})$; bradycardia $(<60$ beats $\left.\cdot \mathrm{min}^{-1}\right)$ is observed in $0-14 \%(\mathrm{I}-\mathrm{H})$ of the patients, and the absolute values of the increase or decrease in heart rate are greater with halothane than with isoflurane. ${ }^{31}$ We have no explanation for these puzzling facts. Nevertheless, they show that succinylcholine, by itself, is well tolerated in healthy nonatropinized children adequately anaesthetized with isoflurane. In other words, some underrated factors (failure to diagnose diseases or abnormalities contraindicating the use of succinylcholine, use of dangerous combinations of drugs, hypoxia, hypercapnia, and vagal reflexes) contribute to many of the undesirable reactions generally attributed to succinylcholine. Normally, an easy and uneventful rapid-sequence tracheal intubation depends on five important factors: (1) technical skill, (2) depth of anaesthesia, (3) neurovegetative stability, (4) degree of muscular relaxation, and (5) length of apnoea. Teaching and clinical research in anaesthesia have emphasized the importance of muscular relaxation. However, it seems evident that a smooth and gentle laryngoscopy is probably less reflexogenic than an abrupt and forceful one. As far as barbiturates may enhance the cardioaccelerating action of atropine, by central inhibition of cardiac vagal neurones, ${ }^{32}$ a higher dose of thiopentone $\left(6-8 \mathrm{mg} \cdot \mathrm{kg}^{-1}\right)$, when not contraindicated, 
may help to reduce the incidence of succinylcholine bradycardia. ${ }^{33-36}$ A longer period of apnoea may lead to hypoxia and hypercapnia, with a higher release of catecholamines, and higher incidence of cardiac arrhythmias following succinylcholine $i v{ }^{37,38}$ Scientific confirmation of these hypotheses, and investigation of the interplay of the above factors have yet to be done.

Over the years, we have thought that bradycardia may occur after a single dose of succinylcholine $i v$ in nonatropinized children, or after repeated doses in nonatropinized adults and children. It is now clear that a single dose of succinylcholine iv may also cause bradycardia and cardiac asystole in nonatropinized adults who received fentanyl for the induction of anaesthesia. ${ }^{39,40}$ Fentanyl activates central cardioinhibition. ${ }^{41}$ The wise course is to avoid the combination of cholinomimetic drugs with a bolus of succinylcholine in nonatropinized patients irrespective of age. Precurarization with d-tubocurarine $\left(0.07 \mathrm{mg} \cdot \mathrm{kg}^{-1}\right)$, or pancuronium $\left(0.03 \mathrm{mg} \cdot \mathrm{kg}^{-1}\right)$, seems to prevent bradycardia, after a second dose of succinylcholine $i v$, in adolescents and adults. ${ }^{42}$ However, these doses may cause hypoventilation, anxiety, and discomfort (heavy eyelids, blurred vision, and difficulty in breathing).

Anaesthetists have widely different beliefs on the indications for succinylcholine in paediatric anaesthesia. Some have stopped using it in elective surgery, ${ }^{43,44}$ others continue to use it even during halothane anaesthesia. ${ }^{45}$ Many anaesthetists probably stand between these extremes. Most admit that one must neither discard the baby with the bath water ${ }^{46}$ nor swamp everybody with deceptive labels or fallacious arguments. Recent studies have raised a new and unsettling point: higher doses of succinylcholine iv $\left(2 \mathrm{mg} \cdot \mathrm{kg}^{-1}\right.$ in children, $3 \mathrm{mg} \cdot \mathrm{kg}^{-1}$ in neonates) could reduce the incidence of difficult intubation, jaw stiffness, and incorrect diagnosis of masseter spasm, even during halothane anaesthesia. ${ }^{45,47-49}$ of course any contraindication must be eliminated, including the possibility of neuromuscular disease (family and personal history, physical examination), before using succinylcholine. ${ }^{43,44}$

In conclusion, atropine and succinylcholine continue to defy our intelligence, common-sense, and imagination. Whether future efforts will be focused on the understanding of their complex effects or on the search for better substitutes remains to be seen. Perhaps we will use a "cardio-elective" $\mathrm{M}_{2}$-antagonist without the side-effects of atropine-like drugs. ${ }^{50}$ Perhaps we will benefit by the discovery of the "perfect muscle relaxant." Anyway, our research and teaching efforts should extend into the assessment of the overall cost-effectiveness of each new drug or procedure. ${ }^{51}$ Meanwhile, let us keep in mind three very simple precautions: (1) the first contraindication to any drug or dosage is the lack of indication; (2) have atropine and succinylcholine immediately available, when not contraindicated; (3) when in doubt, avoid succinylcholine first.

\section{Atropine et succinyl- choline: croyances et controverses en anesthésie pédiatrique}

L'atropine fut isolée en 1831.' Un siècle et soixante-trois ans plus tard, notre compréhension de sa pharmacologie complexe reste encore inachevée. La succinylcholine a été introduite en clinique en 1951. ${ }^{2}$ Quarante-trois ans plus tard, nous n'expliquons pas adéquatement ses méchanismes d'action au niveau de la jonction neuromusculaire, la dualité de son blocage myoneural et la diversité de ses effets neurovégétatifs. Linformation disponible à propos de la physiologie neuromusculaire, de la transmission neurohumorale et de l'emplacement des différents soustypes de récepteurs cholinergiques reste encore fragmentaire. ${ }^{3}$ Dans un tel contexte, il est normal que les anticholinergiques et la succinylcholine continuent de susciter des controverses en anesthésie pédiatrique.

L'atropine produit deux effets contraires sur la fréquence cardiaque, selon la dose utilisée: a) ralentissement, après $1,8 \mu \mathrm{g} \cdot \mathrm{kg}^{-1} i v$ chez les adultes, ou 3,6 $\mu \mathrm{g} \cdot \mathrm{kg}^{-1}$ chez les nourrisons et les enfants; b) accélération, après un minimum de $7,2-14,3 \mu \mathrm{g} \cdot \mathrm{kg}^{-1}$ iv aussi bien chez les nourrissons que chez les enfants et les adultes. ${ }^{4}$ Il fut longtemps admis que l'atropine et la scopolamine produisaient leur cardio-inhibition par stimulation centrale du vague. ${ }^{5}$ Cependant, le ralentissement du pouls est également possible avec des antimuscariniques qui ne traversent pas faciliement la barrière hémato-encéphalique (méthylbromure d'atropine, glycopyrrolate, pirenzépine). 1,6,7 Étant donné qu'une petite dose de pirenzépine, un $\mathbf{M}_{1}$-antagoniste, peut abolir la cardioinhibition de l'atropine, il faut aussi admettre que l'atropine et ses congénères puissent ralentir le coeur en bloquant des auto-récepteurs périphériques de type $M_{1}$ qui servent habituellement à inhiber, ou à graduer, la libération d'acétylcholine. ${ }^{7}$ La cardio-accélération, elle, résulte du blocage de récepteurs $M_{2}$ situés dans le noeud sino-auriculaire. ${ }^{1}$ L'atropine par voie intraveineuse est fréquemment utilisée en anesthésie pédiatrique dans le 
but de 1) prévenir et traiter la dépression myocardique (bradycardie et hypotension) causée par l'halothane, (2) prévenir et traiter la cardio-inhibition associée aux narcotiques puissants (fentanyl et congénères), 3) réduire l'incidence du réflexe oculocardiaque et d'autres réflexes trigéminocardiaques, 4) réduire l'incidence de bradycardies causées par les manipulations des voies aériennes (laryngoscopie, intubation) et par la stimulation viscérale, 5) contrecarrer les effets muscariniques de la néostigmine et de l'édrophonium, 6) réduire les sécréations lors de la chirurgie oro-pharyngée ou de l'usage de la kétamine, et 7) prévenir la bradycardie et les bradyarythmies secondaires à l'injection iv de succinylcholine. Dans toutes ces situations, il est préférable d'utiliser l'atropine $i v$ pour prévenir plutôt que pour traiter les conséquences de la stimulation parasympathique. En effet, si l'atropine est administrée en pleine phase de stimulation vagale, elle risque de convertir une bradycardie sinusale en extrasystoles ventriculaires ou en bigéminisme. ${ }^{8}$ En plus, si l'atropine n'est donnée que durant la bradycardie, particulièrement chez les nourrissons, le débit cardiaque étant diminué, l'effet cardio-accélérateur de l'atropine sera retardé. ${ }^{9}$

Certains auteurs croient que la dose d'atropine (im ou iv) pour les bébés et pour les enfants est de $0,02 \mathrm{mg} \cdot \mathrm{kg}^{-1}$ et accentuent que la " dose minima " pour les nourrissons de moins d'un an est $0,1 \mathrm{mg}$. ${ }^{10,11}$ Le danger de cette "simplification" est illustré par le rapport clinique dun nouveau-né qui a souffert une intoxication (léthargie, opisthotonos, convulsions, respiration périodique, pupilles dilatées et réfractaires à la lumière, peau et muqueuses sèches, rétention urinaire) après avoir reçu deux " doses minima " d'atropine $\left(0,09 \mathrm{mg} \cdot \mathrm{kg}^{-1} i v\right)$ sur une période de 5 heures. ${ }^{12}$ S'il est vrai que plusieurs enfants ont survécu à d'énormes surdosages accidentels (16-40 $\mathrm{mg} \cdot \mathrm{kg}^{-1}$ d'atropine, par voie orale), ${ }^{11,13,14}$ il n'est pas moins vrai que Gillick ${ }^{12}$ rehausse le fait que des décès par intoxication à l'atropine ont été rapportés chez des enfants qui n'ont reçu que $0,05 \mathrm{mg} \cdot \mathrm{kg}^{-1}$. D'assez larges variations individuelles ont été rapportées et dans la pharmacocinétique ${ }^{15}$ et dans la pharmacodynamie de l'atropine. ${ }^{4}$ Des différences par rapport à l'âge ont été mises en évidence par Dauchot et Gravenstein ${ }^{4}$ qui utilisèrent des doses cumulatives d'atropine iv chez des patients éveillés. Les rythmes cardiaques d'origine auriculaire ou nodale, après $1,8-3,6 \mu \mathrm{g} \cdot \mathrm{kg}^{-1}$ d'atropine $i$, sont plus fréquents chez les enfants, tandis que la dissociation A$V$ est l'arythmie cardiaque la plus fréquente chez les adultes qui ont reçu $3,6-7,2 \mu \mathrm{g} \cdot \mathrm{kg}^{-1}$ d'atropine $i{ }^{4}$ Des études sur la pharmacocinétique de l'atropine ont montré que les bébés (jusqu'à deux ans) ont un volume de distribution augmenté $\left(3,2 \mathrm{~L} \cdot \mathrm{kg}^{-1}\right)$ et une demi-vie d'élimination allongée $(6,9 \mathrm{~h})$, après $0,01 \mathrm{mg} \cdot \mathrm{kg}^{-1}$ d'atropine $i v$, par rapport aux enfants plus âgé $\left(1,3 \mathrm{~L} \cdot \mathrm{kg}^{-1}\right.$ et 2,5 $h$, respectivement) et par rapport aux adultes (1,6-3 $\mathrm{L} \cdot \mathrm{kg}^{-1}$ et $3-4 \mathrm{~h}$, respectivement, selon la dose utilisée: $\left.0,01-0,03 \mathrm{mg} \cdot \mathrm{kg}^{-1}\right) .{ }^{15-17}$ Des différences d'ordre ethnique ont été étudiées chez des adultes et non pas chez des enfants. Les Américains blancs semblent plus aptes à développer une bradycardie, après une petite dose d'atropine, que les Américains noirs. ${ }^{18}$ Les Vendas SudAfricains semblent plus sensibles à l'effet cardio-inhibiteur de l'atropine que les Sud-Africains blancs. ${ }^{19}$ Les Chinois semblent beaucoup plus sensibles à l'effet cardioaccélérateur de l'atropine que les Américains blancs. ${ }^{20}$ L'halothane, comparativement à l'enflurane, semble faciliter la cardio-accélération des anticholinergiques, chez les enfants. ${ }^{21}$ Finalement, il faudra se rappeler que plusieurs $\mathrm{H}_{1}$-antihistaminiques, les phénothiazines, les antidépresseurs tricycliques, la gallamine et le pancuronium ont des effets antimuscariniques et, de ce fait, peuvent justifier la diminution de la dose, ou l'omission, de l'atropine.

Dans ce numéro, Shorten et al. ${ }^{22}$ montrent qu'il ne faut pas administrer plus que $10 \mu \mathrm{g} \cdot \mathrm{kg}^{-1}$ d'atropine aux enfants de six à seize ans qui reçoivent de la thiopentone $\left(5 \mathrm{mg} \cdot \mathrm{kg}^{-1}\right)$ et de la succinylcholine iv $(1,5$ $\left.\mathrm{mg} \cdot \mathrm{kg}^{-1}\right)$. Quelqu'un pourrait bien argumenter que cette étude n'apporte rien de nouveau, ne fait que confirmer les suggestions réitérées par d'autres depuis $1963,{ }^{23-25}$ et inclut des adolescents dans un petit échantillonnage. Certains anesthésistes pourront bien se demander si les résultats de l'étude de Shorten et al. justifient le changement de leurs habitudes. D'autres admettront, probablement, que ce quil y a d'irritant dans cette étude c'est qu'ils n'ont pas songé à l'exécuter eux-mêmes. Il y a près de vingt ans, dans notre hôpital, nous avons réexaminée le problème de la " dose efficace minima " d'atropine iv parce que nous avions remarqué que la dose de 0,02 $\mathrm{mg} \cdot \mathrm{kg}^{-1}$ était souvent excessive dans la mesure où elle produisait une haute incidence d'effets indésirables menant à l'inconfort postopératoire (tachycardie, bouche sèche, soif, érythème facial, cervical et thoracique, hyperthermie, rétention urinaire, difficulté d'accomodation visuelle et agitation). Ces effets indésirables étaient particulièrement évidents chez les bébés qui avaient reçu deux doses d'atropine iv $\left(0,04 \mathrm{mg} \cdot \mathrm{kg}^{-1}\right)$ au cours d'une même anesthésie (une dose préanesthésique plus une dose lors de la décurarisation, par exemple). Par conséquent, nous avons décidé de titrer la réponse chronotropique de chaque enfant en utilisant des doses cumulatives de $0,01 \mathrm{mg} \cdot \mathrm{kg}^{-1}$ d'atropine $\dot{w}$. Ça n'a pas pris longtemps pour vérifier que l'injection rapide de $0,01 \mathrm{mg} \cdot \mathrm{kg}^{-1} \mathrm{~d}$ 'atropine iv était remarquablement efficace pour produire la cardio-accélération, et pour réduire ou abolir les effets cardio-inhibiteurs de l'halothane, de la succinylcholine iv 
(1-1,5 $\mathrm{mg} \cdot \mathrm{kg}^{-1}$, après $6-8 \mathrm{mg} \cdot \mathrm{kg}^{-1}$ de thiopentone) et des réflexes d'origine vagale, tout en permettant de réduire considérablement l'inconfort postopératoire. Depuis lors, mise à part la décurarisation, il est extrêmement rare que nous ayons besoin d'utiliser plus que $10 \mu \mathrm{g} \cdot \mathrm{kg}^{-1}$ d'atropine $i v$. D'ailleurs, des courbes de dose-réponse obtenues avec des doses cumulatives d'atropine iv confirment que la "dose efficace minima " pour produire la cardio-accélération se situe quelque part entre 7,2 et 14,3 $\mu \mathrm{g} \cdot \mathrm{kg}^{-1}$ (pour les nourrissons, les enfants et les adultes également). ${ }^{4}$ Nous croyons donc que Shorten $e t$ al. ${ }^{22}$ ont trouvé une dose raisonnable d'atropine $i v$ pour prévenir la bradycardie de la succinylcholine.

La bradycardie (moins de 100 battements $\cdot \mathrm{min}^{-1}$ jusqu’à la première année de vie, 98 battements $\cdot \mathrm{min}^{-1}$ jusqu'à deux ou trois ans, $60-65$ battements $\cdot \mathrm{min}^{-1}$ chez les enfants plus âgés) est souvent un signe d'hypoxie, d'hypotension sévère, ou de surdosage d'anesthésique qui peut mener à l'arrêt circulatoire particulièrement chez les bébés. L'incapacité de reconnaître la présence et la cause de la bradycardie et l'incapacité d'éviter ou de renverser la tendance vers la bradycardie seraient plus fréquentes chez les novices que chez les anesthésistes qui ont de l'expérience en anesthésie pédiatrique. ${ }^{26,27}$

La succinylcholine, lorsque non contre-indiquée, est le curarisant de choix pour l'intubation en séquence rapide, le laryngospasme, la réintubation d'urgence et l'administration intramusculaire. ${ }^{28-30}$ Contenant deux molécules d'acétylcholine rassemblées bout à bout, la succinylcholine peut reproduire tous les effets muscariniques et nicotiniques de l'acétylcholine. Chez des enfants non atropinisés (âgés de trois mois jusqu’à sept ans), bien ventilés et anesthésiés à l'halothane $(\mathrm{H}), 2,5 \%$ dans l'oxygène, ou à l'soflurane (I), 3,5\% dans l'oxygène, une injection rapide de $1,5 \mathrm{mg} \cdot \mathrm{kg}^{-1}$ de succinylcholine iv produit trois effets différents et imprévisibles sur la fréquence cardiaque: a) augmentation (incidence de $66 \%$ avec $\mathrm{H}, 88 \%$ avec I), b) aucun changement $(0-7 \%, \mathrm{I}-\mathrm{H})$, ou c) diminution $(12-25 \%$, I- $\mathrm{H})$; la bradycardie $(<60$ battements $\left.\cdot \min ^{-1}\right)$ n'est observée que dans $0-14 \%$ des cas (I-H) et les valeurs absolues de l'augmentation ou de la diminution de la fréquence cardiaque sont plus élevées avec l'halothane qu'avec l'isoflurane. ${ }^{31}$ Nous n'avons pas d'explication pour ces faits plutôt troublants. Néanmoins, ces faits nous montrent que la succinylcholine en soi est assez bien tolérée par des enfants normaux, non atropinisés, et bien anesthésiés à l'isoflurane. En d'autres termes, des facteurs souvent sous-évalués (incapacité de diagnostiquer des maladies ou des anomalies qui contreindiquent l'usage de la succinylcholine, utilisation d'associations médicamenteuses plutôt dangereuses, hypoxie, hypercapnie, reflexes d'origine vagale) contribuent à l'incidence de réactions indésirables généralement attribuées à la succinylcholine. Normalement, la réussite et linnocuité de lintubation en séquence rapide dépendent de cinq facteurs importants: 1) dextérité technique, 2) profondeur de l'anesthésie, 3) stabilité neurovégétative, 4) niveau de curarisation, et 5) durée de l'apnée. L'enseignement et la recherche en anesthésie ont surtout mis l'accent sur l'importance de la curarisation. Cependant, il semble évident qu'une l'aryngoscopie douce et délicate est moins réflexogène qu'une laryngoscopie brusque et énergique. Dans la mesure où les barbituriques facilitent la cardioaccélération de l'atropine, en inhibant le centre vagal, ${ }^{32}$ une dose un peu plus grande de thiopentone (6-8 $\left.\mathrm{mg} \cdot \mathrm{kg}^{-1}\right)$, si elle n'est pas contre-indiquée peut aider à réduire l'incidence de bradycardies reliées à la succinylcholine. ${ }^{33-36}$ Une période d'apnée un peu trop longue peut faciliter l'hypoxie et l'hypercapnie, augmenter la libération de catécholamines, et contribuer à une haute incidence d'arythmies cardiaques attribuées à la succinylcholine. ${ }^{37,38}$ La confirmation scientifique de ces hypothèses et l'investigation de l'interinfluence des facteurs sus-mentionnés restent encore à faire.

Pendant des années, nous avons cru que la bradycardie peut résulter d'une dose unique de succinylcholine iv chez les enfants non atropinisés, ou de doses répétées chez les adultes et les enfants non atropinisés. Il est maintenant bien clair qu'une dose unique de succinylcholine iv peut également déclencher une bradycardie, ou une asystolie, chez des adultes non atropinisés qui ont reçu du fentanyl lors de l'induction de l'anesthésie. ${ }^{39-40}$ Le fentanyl active la cardio-inhibition d'origine centrale. ${ }^{41}$ Il est donc prudent de ne pas associer des agents cholinomimétiques avec la succinylcholine iv chez les patients de tout âge qui n'ont pas reçu d'atropine. La précurarisation avec la $d$ tubocurarine $\left(0,07 \mathrm{mg} \cdot \mathrm{kg}^{-1}\right)$, ou avec le pancuronium $\left(0,03 \mathrm{mg} \cdot \mathrm{kg}^{-1}\right)$, semble prévenir la bradycardie due à une deuxième dose de succinylcholine $i v$ chez les adolescents et les adults. ${ }^{42}$ Cependant, ces doses de nondépolarisants peuvent induire de l'hypoventilation, de l'anxiété et de linconfort (paupières lourdes, vue brouillée, difficulté à respirer).

Les anesthésistes ont des opinions fort divergentes quant aux indications de la succinylcholine en anesthésie pédiatrique. Certains ont tendance à la proscrire de la chirurgie élective ${ }^{43,44}$ alors que d'autres continuent de luutiliser même avec de l'halothane. ${ }^{45}$ Plusieurs anesthésiologistes restent, probablement, entre ces extrêmes. La plupart éventuellement conviendra qu'il ne faut pas rejeter le bébé avec l'eau de son bain ${ }^{46}$ ni essayer de noyer tout le monde avec des étiquettes trompeuses ou des arguments fallacieux. Des études récentes soulèvent un point perturbateur: des doses plus fortes de succinylcholine (2 $\mathrm{mg} \cdot \mathrm{kg}^{-1}$ chez les enfants, $3 \mathrm{mg} \cdot \mathrm{kg}^{-1}$ chez les nouveaunés) pourraient réduire lincidence d'intubations difficiles, 
de rigidité mandibulaire, et de faux diagnostics de spasme des masséters, même avec de l'halothane. ${ }^{45,47-49}$ Évidemment, il faut essayer d'éliminer toute contra-indication, sans oublier la possibilité de maladie neuromusculaire (histoire personnelle et familiale, examen physique), avant d'utiliser la succinylcholine. ${ }^{43,44}$

En conclusion, l'atropine et la succinylcholine continuent à défier notre intelligence, notre bon sens et notre imagination. Il reste à savoir si nos efforts seront polarisés sur la compréhension de leurs pharmacologies complexes ou sur la recherche de meilleurs remplaçants. Peut-être utiliserons-nous un $\mathrm{M}_{2}$-antagoniste " cardio-sélectif » qui n'aura pas les effets indésirables des atropinomimétiques. ${ }^{50}$ Peut-être bénéficierons-nous de la découverte du " curarisant parfait ». De toute façon, nos efforts en recherche et dans l'enseignement devront bien s'étendre jusqu'à l'évaluation du rapport coût/efficacité de chaque nouveau médicament ou nouvelle procédure. ${ }^{51} \mathrm{En}$ attendant, gardons à l'esprit trois précautions assez simples: 1) la première contre-indication de nimporte quel médicament, ou de nimporte quel dosage, est le manque d'indication; 2) ayons l'atropine et la succinylcholine sous la main, si elles ne sont pas contre-indiquées; 3) dans le doute, évitons d'abord la succinylcholine.

\section{References}

1 Brown JH. Atropine, scopolamine, and related antimuscarinic drugs. In: Gilman AG, Rall TW, Nies AS, Taylor P (Eds.). Goodman and Gilman's The Pharmacological Basis of Therapeutics, 8th ed., New York: Pergamon Press 1990; 150-65.

2 Brücke H, Ginzel KH, Klupp H, Pfaffenschlager F, Werner $G$. Bis-cholinester von dicarbonsäuren als muskelrelaxantien in der narkose. Wien Klin Wochenschr 1951; 63 : 464-6.

3 Lefkowitz RJ, Hoffman BB, Taylor P. Neurohumoral transmission: the autonomic and somatic nervous systems. In: Gilman AG, Rall TW, Nies AS, Taylor P (Eds.). Goodman and Gilman's The Pharmacological Basis of Therapeutics, 8th ed., New York: Pergamon Press 1990; 84-121.

4 Dauchot P, Gravenstein JS. Effects of atropine on the electrocardiogram in different age groups. Clin Pharmacol Ther 1971; 12: 274-80.

5 Heinekamp WJR. The central influence of atropine and hyoscine on the heart rate. J Lab Clin Med 1922; 8: 104-11.

6 Kottmeier CA, Gravenstein JS. The parasympathomimetic activity of atropine and atropine methylbromide. Aresthesiology 1968; 29: 1123-33.

7 Wellstein A, Pitschner HF. Complex dose-response curves of atropine in man explained by different functions of $\mathrm{M}_{\mathrm{l}^{-}}$ and $\mathrm{M}_{2}$-cholinoceptors. Naunyn Schmiedbergs Arch Pharmacol 1988; 338: 19-27.
8 Blanc VF. Trigeminocardiac reflexes (Editorial). Can J Anaesth 1991; 38: 696-9.

9 Zimmerman G, Steward DJ. Bradycardia delays the onset of intravenous atropine in infants. Anesthesiology 1986; 65: $320-2$.

10 Steward DG. Manual of Pediatric Anesthesia, 3rd ed., New York. Churchill Livingstone 1990.

11 Steward DI. Psychological preparation and premedication. In: Gregory GA (Ed.). Pediatric Anesthesia, 2nd ed., New York: Churchill Livingstone 1989; 523-38.

12 Gillick $J S$. Atropine toxicity in a neonate. $\mathrm{Br} \mathbf{J}$ Anaesth 1974; 46: 793-4.

13 MacKenzie AL, Pigott JFG. Atropine overdose in three children. Br J Anaesth 1971; 43: 1088-90.

14 Arthurs GJ, Davies $R$. Atropine - a safe drug. Anaesthesia 1980; 35: 1077-9.

15 Adams RG, Verma P, Jackson AJ, Miller RL. Plasma pharmacokinetics of intravenously administered atropine in normal human subjects. J Clin Pharmacol 1982; 22: 477-81.

16 Virtanen $R$, Kanio J, Iisalo EUM, Salo M, Sjövall S. Pharmacokinetic studies on atropine with special reference to age. Acta Anaesthesiol Scand 1982; 26: 297-300.

17 Hinderling PH, Gundert-Remy U, Schmidlin $O$. Integrated pharmacokinetics and pharmacodynamics of atropine in healthy humans. I: Pharmacokinetics. J Pharmac Sci 1985; 74: 703-10.

18 Paskind $H A$. Some differences in response to atropine in white and colored races. J Lab Clin Med 1921; 7: 104-8.

19 Meyer EC, Sommers DK, Schoeman HS, Avenant JC. The effect of atropine on heart-rate: a comparison between two ethnic groups (Letter). Br J Clin Pharmac 1988; 25: 776-7.

20 Zhou $H-H$, Adedoyin A, Wood AJJ. Differing effect of atropine on heart rate in Chinese and white subjects. Clin Pharmacol Ther 1992; 52: 120-4.

21 Samra SK, Cohen PJ. Modification of chronotropic response to anticholinergics by halogenated anaesthetics in children. Can Anaesth Soc J 1980; 27: 540-5.

22 Shorten $G D$, Bissonette B, Hartley E, Nelson W, Carr AS. It is not necessary to administer more than $10 \mu \mathrm{g} \cdot \mathrm{kg}^{-1}$ of atropine to older children before succinylcholine. Can J Anaesth 1995; 42: 8-11.

23 Sagarminaga J, Wynands JE. Atropine and the electrical activity of the heart during induction of anaesthesia in children. Can Anaesth Soc J 1963; 10: 328-41.

24 Blanc $V F$. Anticholinergic premedication for infants and children (the oculocardiac reflex) (Letter). Can Anaesth Soc J 1983; 30: 683-4.

25 Goudsouzian NG. Relaxants in paediatric anaesthesia. Clinics in Anaesthesiology 1985; 3: 539-51.

26 Keenan $R L$, Shapiro JH, Kane FR, Simpson PM. 
Bradycardia during anesthesia in infants: an epidemiologic study. Anesthesiology 1994; 80: 976-80.

27 Morray JP. Implications for subspecialty care of anesthetized children (Editorial). Anesthesiology 1994; 80: 969-71.

28 Bevan DR, Bevan JC, Donati F. Muscle Relaxants in C' nical Anesthesia. Chicago: Year Book Medical Publ Inc., 1988.

29 Goudsouzian NG. Muscle relaxants in children. In: Coté CJ, Ryan JF, Todres ID, Goudsouzian NG (Eds.). A Practice of Anesthesia for Infants and Children, 2nd ed., Philadelphia: WB Saunders Company 1993; 151-70.

30 Flynn PJ. Pharmacokinetics and pharmacodynamics of succinylcholine. Anesthesiology Clinics of North America 1993; 11: 309-24.

31 Lerman J, Robinson S, Willis MM, Schmitt BI, Gregory $G A$. Succinylcholine-induced heart rate changes in children during isoflurane and halothane. Anesthesiology 1983; 59: A443.

32 Inoue $K$, Arndt JO. Efferent vagal discharge and heart rate in response to methohexitone, althesin, ketamine and etomidate in cats. Br J Anaesth 1982; 54: 1105-16.

33 Williams $C H$, Deutsch S, Linde HW, Bullough JW, Dripps $R D$. Effects of intravenously administered succinyldicholine on cardiac rate, rhythm, and arterial blood pressure in anesthetized man. Anesthesiology 1961; 22: 947-54.

34 McIntyre JWR. Succinylcholine-induced bradycardia with reference to methoxyflurane anaesthesia. Can Anaesth Soc J 1962; 9: 408-13.

35 Schoenstadt DA, Whitcher $C E$. Observations on the mechanism of succinyldicholine-induced cardiac arrhythmias. Anesthesiology 1963; 24: 359-62.

36 Viby-Mogensen J, Wisborg $K$, Sørensen $O$. Cardiac effects of atropine and gallamine in patients receiving suxamethonium. Br J Anaesth 1980; 52: 1137-42.

37 McCullough LS, Nigrovic C, Wajskol A, Levin J, Martin $J T$. Release of catecholamines by succinylcholine in man. Anesth Analg 1982; 61: 203 (Abstract).

38 Leiman BC, Katz J, Butler BD. Mechanisms of succinylcholine-induced arrhythmias in hypoxic or hypoxic: hypercarbic dogs. Anesth Analg 1987; 66: 1292-7.

39 Sørensen M, Engbaek J, Viby-Mogensen J, Guldager $H$, Jensen $F M$. Bradycardia and cardiac asystole following a single injection of suxamethonium. Acta Anaesthesiol Scand 1984; 28: 232-5.

40 Inoue $K$, Reichelt $W$. Asystole and bradycardia in adult patients after a single dose of suxamethonium. Acta Anaesthesiol Scand 1986; 30: 571-3.

41 Inoue $K$, Samodelov $L F$, Arndt JO. Fentanyl activates a particular population of vagal efferents which are cardioinhibitory. Naunyn Schmiedbergs Arch Pharmacol 1980; 312: 57-61.

42 Mathias JA, Evans-Prosser CDG, Churchill-Davidson HC. The role of the non-depolarizing drugs in the prevention of suxamethonium bradycardia. Br J Anaesth 1970; 42 : 609-13.

43 Delphin E, Jackson D, Rothstein P. Use of succinylcholine during elective pediatric anesthesia should be reevaluated. Anesth Analg 1987; 66: 1190-2.

44 Rosenberg $H$, Gronert $G A$. Intractable cardiac arrest in children given succinylcholine (Letter). Anesthesiology 1992; 77: 1054.

45 Hannallah RS, Kaplan RF. Jaw relaxation after halothane/succinylcholine sequence in children. Anesthesiology 1994; 81: 99-103.

46 Morell RC, Berman JM, Royster RI, Petrozza PH, Kelly $J S$, Colonna DM. Revised label regarding the use of succinylcholine in children and adolescents: I (Letter). Anesthesiology 1994; 80: 242.

47 Meakin G, McKiernan EP, Morris P, Baker RD Doseresponse curves for suxamethonium in neonates, infants and children. Br J Anaesth 1989; 62: 655-8.

48 Meakin $G$. Underdosage with succinylcholine may lead to incorrect diagnosis of masseter spasm in children (Letter). Anesthesiology 1988; 69: 1025-6.

49 Leary $N P$, Ellis $F R$. Masseteric muscle spasm as a normal response to suxamethonium. $\mathrm{Br} J$ Anaesth 1990; 64: 488-92.

50 Pitschner HF, Schulte B, Schlepper M, Palm D, Wellstein A. AF-DX 116 discriminates heart from gland $\mathrm{M}_{2}$ cholinoceptors in man. Life Sci 1989; 45: 493-8.

51 Antebi $M E$, Ornstein E. Economic considerations for new anesthesia drugs. Anesthesiology Clinics of North America $1994 ; 12: 39-48$. 